\title{
Growth of III-nitride photonic structures on large area silicon substrates
}

J. Li

III-N Technology Inc., 2601 Anderson Avenue, Suite 102, Manhattan, Kansas 66502

J. Y. Lin and H. X. Jiang ${ }^{a}$

Department of Physics, Kansas State University, Manhattan, Kansas 66506-2601

(Received 14 December 2005; accepted 15 March 2006; published online 26 April 2006)

\begin{abstract}
We report on the growth of high quality aluminum nitride (AIN) and gallium nitride $(\mathrm{GaN})$ epilayers on large area (6 in. diameter) silicon (111) substrates by metal organic chemical vapor deposition. We have demonstrated the feasibility of growing crack-free high quality III-nitride photonic structures and devices on 6 inch Si substrates through the fabrication of blue light emitting diodes based upon nitride multiple quantum wells with high performance. The demonstration further enhances the prospects for achieving photonic integrated circuits based upon nitride-on-Si material system. (C) 2006 American Institute of Physics. [DOI: 10.1063/1.2199492]
\end{abstract}

III-nitride wide band gap semiconductors have recently attracted much attention because of their applications in blue/UV optoelectronic devices as well as high power, high temperature electronic devices. ${ }^{1}$ The growth of III-nitride photonic structures on large area $\mathrm{Si}$ substrates presents a unique opportunity for the integration of photonic devices with standard Si electronics and development of photonic integrated circuits (PICs) for a wide range of applications. The unique properties of III-nitrides may allow the creation of PICs with unprecedented properties and functions. ${ }^{2}$ PIC technology eventually would allow the integration of arrays of thousands of optical circuit elements such as emitters, detectors, waveguides, switches, etc., on a single chip. Together with their two-dimensional array nature, III-nitride PICs may open up many important applications in the areas of optical communications and medical diagnosis. Recent work has also revealed that $\mathrm{Al}_{x} \mathrm{Ga}_{1-x} \mathrm{~N}$ is a promising material system for optical communication applications in the $1.55 \mu \mathrm{m}$ wavelength window due to its ability of providing high damage threshold and controllable indices through heterostructure engineering and carrier injection. ${ }^{3-7}$

Due to the great potential of the nitride-on-Si material system, intensive research efforts have been dedicated to the optimization of epitaxial growth processes and stress control methods over the past decade. ${ }^{8-24}$ Apart from a high dislocation density in the grown nitride films due to the large lattice mismatch, another main challenge is managing the stress induced by the coefficient of thermal expansion mismatch that leads to cracks in epilayers thicker than $1 \mu \mathrm{m} .{ }^{8}$

Several approaches have been previously employed to manage stress and minimize the cracks, such as the insertion of $\mathrm{AlGaN} / \mathrm{GaN}$ superlattice structure, ${ }^{9,18,21,23}$ employing variations of $\mathrm{AlN}$ nucleation and $\mathrm{Al}_{x} \mathrm{Ga}_{1-x} \mathrm{~N}$ graded layers, $8,10,13,19,23$ and epitaxial lateral overgrowth using patterned $\mathrm{Si}$ substrate with $\mathrm{Si}_{x} \mathrm{~N}_{1-x}$ or $\mathrm{SiO}_{2}$ masks. ${ }^{15,22}$ In terms of device performance, $\mathrm{Si}_{x} \mathrm{~N}_{1-x}$ in situ masking and subsequent lateral overgrowth technique seem to provide promising results. ${ }^{22}$ All of the previously demonstrated epitaxial growth of nitride photonic structures on $\mathrm{Si}$ was for small $\mathrm{Si}$ substrates $(<2$ in. in diameter). Although nitride based heterojunction field effect transistors have been successfully

\footnotetext{
${ }^{a)}$ Electronic mail: jiang@phys.ksu.edu
}

fabricated on 4 in. Si substrates, ${ }^{20}$ there have been no previous demonstrations of the growth of nitride materials and devices on Si substrates with diameters larger than 4 inch.

For the growth of nitride materials on large area Si substrates, problems associated with cracks and bowing are expected to be much more severe because the demand on the temperature uniformity and mechanical strength over the whole wafer is higher. Previous studies have indicated that due to its smaller lattice constant, it is possible for AlN nucleation layer to induce compressive strain on the subsequent GaN layers, thereby supporting the growth of crackfree thicker GaN layers by counterbalancing the thermally induced tensile strains. ${ }^{19}$ In this letter, we report on the growth of high quality AlN and GaN epilayers on large area (6 in. diameter) Si (111) substrates by metal organic chemical vapor deposition (MOCVD). By exploiting the combination of a high quality AlN epilayer and a thin $\mathrm{Al}_{x} \mathrm{Ga}_{1-x} \mathrm{~N}$ graded layer on $\mathrm{Si}$ as a template, we demonstrate that the growth of III-nitride photonic structures on large area $\mathrm{Si}$ substrates is possible. We have grown InGaN/GaN multiple quantum well (MQW) blue LED structures on 6 in. Si (111) substrates and the fabricated devices exhibited high performance.

A commercial low pressure MOCVD reactor (commissioned by Thomas Swan Scientific Equipment Ltd.) was used for the epitaxial growth. The original reactor was designed for the simultaneous growth of seven pieces of $2 \mathrm{in}$. wafers. In order to carry out III-nitride epitaxial growth on 6 inch $\mathrm{Si}$ substrates, we designed a $1 \times 6$ inch $^{2} \mathrm{SiC}$ coated graphite susceptor. The total gas flow rate was about $251 / \mathrm{min}$. Single crystalline Si wafers with $\langle 111\rangle$ orientation were used as substrates. No extra treatments were employed before transferring Si substrates into the reactor. For the growth of AlN on 6 inch Si substrate, the temperature profile of the susceptor has to be carefully monitored to insure good uniformity across the entire 6 inch area. The Si wafer was first heated up to $1100{ }^{\circ} \mathrm{C}$ for $15 \mathrm{~min}$ to clean the surface. A lowtemperature AlN nucleation layer was deposited at $500{ }^{\circ} \mathrm{C}$ prior to the deposition of the high quality AlN epilayer. The AlN epilayers were grown at $1050{ }^{\circ} \mathrm{C}$ on Si and $1300{ }^{\circ} \mathrm{C}$ on sapphire. The thickness uniformity across the whole wafer was within $10 \%$ as measured by the reflectance spectra. The optical qualities of AlN epilayers were characterized by a 


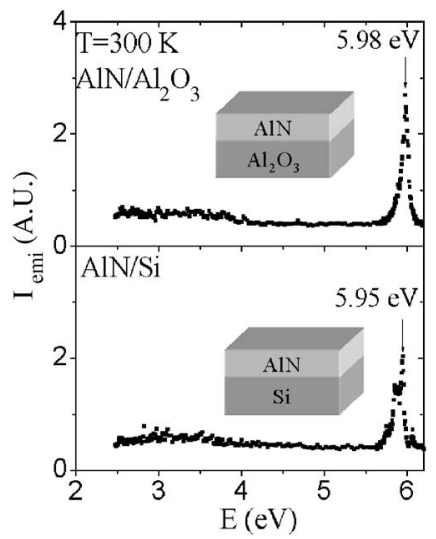

FIG. 1. Room temperature PL spectra of AlN epilayers grown on (a) 2 in. sapphire and (b) 6 in. Si substrates.

specially designed deep UV photoluminescence (PL) spectroscopy system, which consists of a frequency quadrupled $100 \mathrm{f} \mathrm{(76} \mathrm{MHz} \mathrm{repetition)} \mathrm{Ti:sapphire} \mathrm{laser} \mathrm{together} \mathrm{with} \mathrm{a}$ $1.3 \mathrm{~m}$ monochromator and streak camera, providing an average excitation power of about $3 \mathrm{~mW}$ at $196 \mathrm{~nm}$.

Figure 1 compares the room temperature PL spectra of AlN epilayers grown on 2 in. sapphire and 6 in. Si substrates. The PL spectral line shapes from both samples are similar. The PL results show that AlN epilayers grown on $\mathrm{Si}$ exhibit predominantly the band-edge PL emission-implying high optical quality. Transmission electron microscopy (TEM) and high-resolution x-ray diffraction (XRD) studies provide evidence that the final resultant stress is compressive in our AlN epilayers grown on sapphires. ${ }^{25}$ The resultant stress in AlN epilayers grown on $\mathrm{Si}$ is generally expected to be tensile. This indeed seems to be the case by noting that the PL spectral peak position in AlN deposited on Si is redshifted with respect to that in AlN deposited on sapphire (5.95 $\mathrm{eV}$ for AlN on Si versus $5.98 \mathrm{eV}$ for AlN on sapphire). However, no cracks were observable in the grown AlN epilayers. We previously attribute the dominant emission peak in high quality AlN epilayers at room temperature to the recombination of free excitons, which have a binding energy of about $80 \mathrm{meV}$ in $\mathrm{AlN} .^{26,27}$

GaN epilayers were grown on AlN epilayer/sapphire template. By inserting a thin $\mathrm{Al}_{x} \mathrm{Ga}_{1-x} \mathrm{~N}$ graded layer $(\sim 50 \mathrm{~nm})$ in between the AlN epilayer template and the subsequent $\mathrm{GaN}$ epilayer, cracks and wafer bowing were eliminated. Figure 2(a) shows the XRD $\theta / 2 \theta$ curve of a $2 \mu \mathrm{m}$ $\mathrm{GaN}$ epilayer deposited on the AlN epilayer $(0.5 \mu \mathrm{m}) / \mathrm{Si}$ substrate, and the result shows good crystalline quality of the $\mathrm{GaN}$ epilayer. We can see the diffraction peaks from Si (111) at $28.367^{\circ}, \mathrm{GaN}(0002)$ at $34.547^{\circ}$, and $\mathrm{AlN}(0002)$ at $36.027^{\circ}$ with the full width at half maximum (FWHM) for $\mathrm{GaN}$ of about $180 \mathrm{arcsec}$. The XRD rocking curve of the (0002) peak of GaN was measured in different locations (at the center and also at the edge of the wafer), which shows a variation within $2 \%$ with a typical FWHM of about 565 arc sec, as shown in Fig. 2(b). This is among the best results for $\mathrm{GaN}$ epilayers grown on $\mathrm{Si}$ substrates. The rootmean-square (rms) deviation of the sample surface measured by atomic force microscopy (AFM) is only $0.8 \mathrm{~nm}$ for a 2 $\times 2 \mu \mathrm{m}^{2}$ scan, indicating a very good surface morphology. The sheet resistance measurements revealed a $2 \%$ variation in sample resistivity across the entire 6 in. wafer. The benefits of inserting AlN epitaxial layer as a template for the

Downloaded 12 Jul 2010 to 129.118 .86 .45 . Redistribution subject
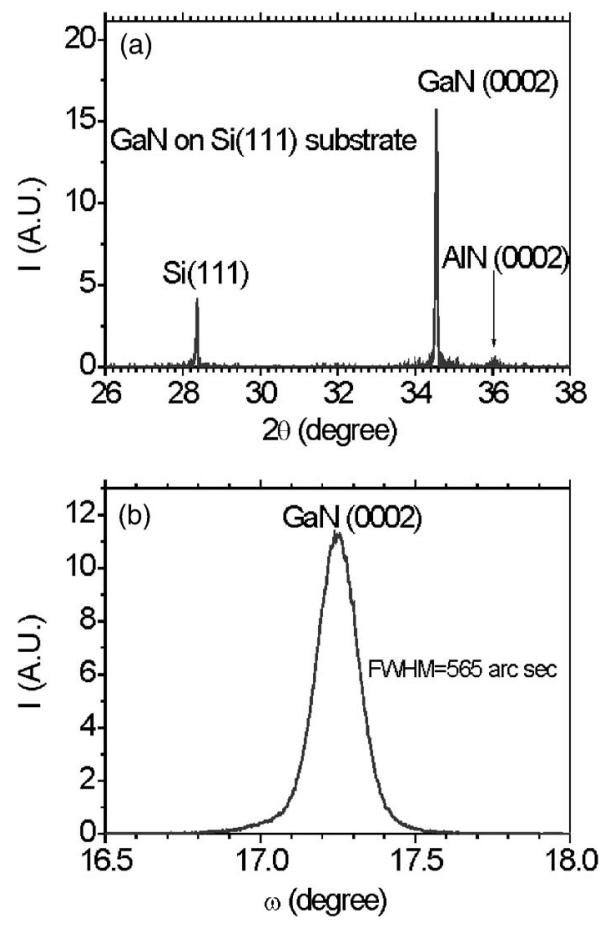

FIG. 2. X-ray diffraction (XRD) spectra of a $2 \mu \mathrm{m}$ thick GaN epilayer grown on AlN epilayer/Si (111) substrate: (a) $\theta / 2 \theta$ scan and (b) rocking curve of the (0002) peak.

growth of subsequent III-nitride device structures on sapphires are well documented. ${ }^{28-30}$ It was previously shown that $\mathrm{GaN}$ epilayers grown on AlN epilayer/sapphire substrate comprise a lower dislocation density compared with the GaN grown directly on sapphire using low-temperature GaN buffer. ${ }^{28}$ It was recently demonstrated that InGaN blue/green LEDs and AlGaN UV LEDs grown on the AlN epilayer/ sapphire substrate exhibited a higher output power and a better thermal stability compared with LEDs grown on sapphire using a low-temperature GaN buffer layer. ${ }^{29}$ This is due to the reduced threading dislocation density in the active layer and higher thermal conductivity of AlN epilayer. ${ }^{28} \mathrm{We}$ believe that the high quality AlN epilayer also acted as an effective dislocation filter for the growth of subsequent device layers deposited on Si substrate.

To further investigate the benefits of using AlN epilayer as a template, we have also grown blue LED structure on 6 in. Si substrates. Prior to the growth of the LED active region, a $0.5 \mu \mathrm{m}$ AlN epilayer was grown on $\mathrm{Si}$ at $1050{ }^{\circ} \mathrm{C}$ as a template. Then a thin $\mathrm{Al}_{x} \mathrm{Ga}_{1-x} \mathrm{~N}$ graded layer was deposited to further minimize cracks and wafer bowing. The subsequent MQW LED structure on AIN epilayer/Si substrate consists of an $\sim 2 \mu \mathrm{m} \mathrm{Si}$ doped $n$-GaN epilayer, 8 periods InGaN/GaN MQW active layer, and a $0.25 \mu \mathrm{m} \mathrm{Mg}$ doped $p$-GaN epilayer.

The characteristics of the fabricated LEDs were also measured. Photolithographic patterning and inductively coupled plasma dry etching were employed to fabricate LED chips $\left(300 \times 300 \mu \mathrm{m}^{2}\right)$. Bilayers of $\mathrm{Ni}(20 \mathrm{~nm}) / \mathrm{Au}$ $(200 \mathrm{~nm})$ and $\mathrm{Al}(300 \mathrm{~nm}) / \mathrm{Ti}(20 \mathrm{~nm})$ were deposited by electron beam evaporation as $p$ - and $n$-type Ohmic contacts, respectively. Figure 3(a) shows the $I-V$ characteristic of the LED on Si. The forward bias voltage at $20 \mathrm{~mA}\left(V_{F}\right)$ is $4.1 \mathrm{~V}$. The reverse leakage current is about $27 \mu \mathrm{A}$ at $-20 \mathrm{~V}$. The forward differential series resistance is about $25 \Omega$. Optical AIP license or copyright; see http://apl.aip.org/apl/copyright.jsp 

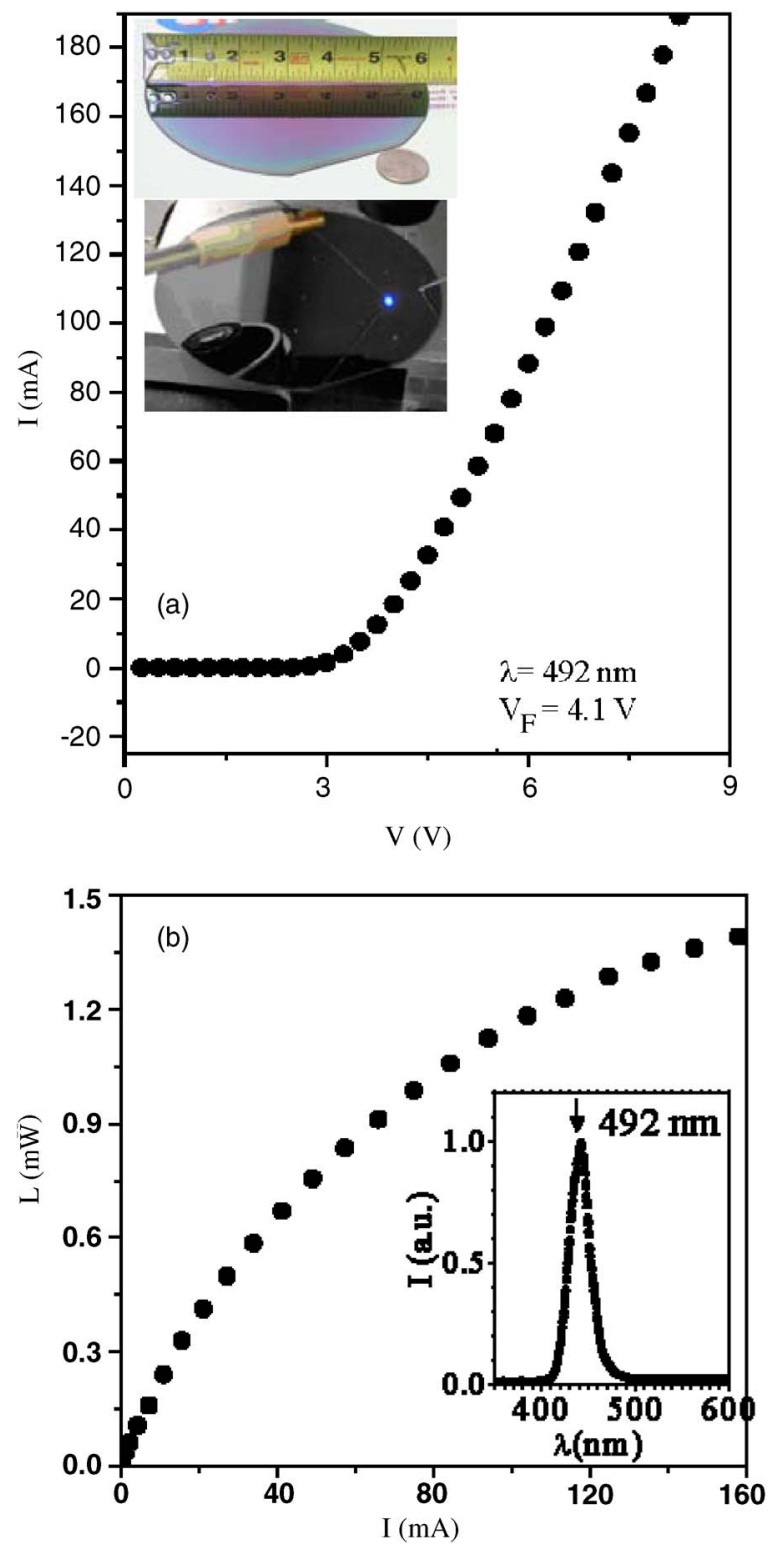

FIG. 3. (Color online) (a) $I-V$ and (b) $L-I$ characteristics of fabricated InGaN/GaN MQW LED grown on AlN epilayer/Si substrate, measured from the top surface of unpackaged bare chips with size of 300 $\times 300 \mu \mathrm{m}^{2}$. The inset in (a) shows the optical microscopy images of an InGaN/GaN MQW blue LED wafer grown on 6 in. Si substrate.

microscopy images of a blue LED wafer grown on a 6 in. $\mathrm{Si}$ substrate are shown in the inset of Fig. 3(a), which show that these LEDs have a good surface morphology and no cracks were observable.

A typical electroluminescence (EL) spectrum of a $492 \mathrm{~nm}$ LED fabricated on Si substrate is also shown in the inset of Fig. 3(b). The FWHM of the emission peak is about $32 \mathrm{~nm}$. We can also see clearly the interference pattern from the EL spectrum indicating again the good surface morphology of the LED wafer. Figure 3(b) shows the $L-I$ characteristics (optical power output as a function of applied current) of the LED fabricated on Si. The optical power output is about $0.35 \mathrm{~mW}$ at $20 \mathrm{~mA}$. Since the optical power was measured from the top surface of unpackaged LED chips, the final output power of the packaged LEDs is expected to be higher. This is so far the best performance achieved for nitride LEDs grown on unpatterned Si substrates.
In summary, we have demonstrated the feasibility for growing high quality III-nitride photonic structures and devices on large area (6 in. diameter) $\mathrm{Si}$ (111) substrates by MOCVD. By employing high quality AlN epilayer in combination with a thin $\mathrm{AlGaN}$ graded layer as a template, we have obtained crack-free GaN epilayers and high performance InGaN/GaN MQW LED structures with a total thickness exceeding $3 \mu \mathrm{m}$. The demonstration further enhances the prospects for achieving photonic integrated circuits based upon nitride-on-Si material system.

The research is supported by NSF Grant Nos. DMI0450314 and DMR-0203373.

${ }^{1}$ H. Morkoç, Nitride Semiconductors and Devices (Springer, Berlin, 1999). ${ }^{2}$ H. X. Jiang and J. Y. Lin, CRC Crit. Rev. Solid State Mater. Sci. 28, 131 (2003).

${ }^{3}$ R. Hui, S. Taherion, Y. Wan, J. Li, S. X. Jin, J. Y. Lin, and H. X. Jiang, Appl. Phys. Lett. 82, 1326 (2003).

${ }^{4}$ R. Hui, Y. Wan, J. Li, S. X. Jin, J. Y. Lin, and H. X. Jiang, IEEE J. Quantum Electron. 41, 100 (2005).

${ }^{5}$ R. Geiss, A. Chowdhury, C. M. Staus, H. M. Ng, S. S. Park, and J. Y. Han, Appl. Phys. Lett. 87132107 (2005).

${ }^{6}$ R. G. Wilson, R. N. Schwartz, C. R. Abernathy, S. J. Pearton, N. Newman, M. Rubin, T. Fu, and J. M. Zavada, Appl. Phys. Lett. 65, 992 (1994).

${ }^{7}$ M. Thaik, U. Hömmerich, R. N. Schwartz, R. G. Wilson, and J. M. Zavada, Appl. Phys. Lett. 71, 2641 (1992).

${ }^{8}$ A. Watanabe, T. Takeuchi, K. Hirosawa, H. Amano, K. Hiramatsu, and I. Akasaki, J. Cryst. Growth 128, 391 (1993).

${ }^{9}$ H. Ishigawa, G. Y. Zhao, N. Nakada, T. Egawa, T. Soga, T. Jimbo, and M. Umeno, Phys. Status Solidi A 176, 599 (1999).

${ }^{10}$ C. A. Tran, A. Osinski, R. F. Karlicek, and I. Berishev, Appl. Phys. Lett. 75, 1494 (1999)

${ }^{11}$ J. W. Yang, A. Lunev, G. Simin, A. Chitnis, M. Shatalov, M. A. Khan, J. E. Van Nostrand, and R. Gaska, Appl. Phys. Lett. 76, 273 (2000).

${ }^{12} \mathrm{H}$. Lahrèche, P. Vennéguès, O. Totterau, M. Laügt, P. Lorenzini, M. Leroux, B. Beaumont, and P. Gibart, J. Cryst. Growth 217, 13 (2000).

${ }^{13}$ J. Han, K. E. Waldrip, S. R. Lee, J. J. Figiel, S. J. Hearne, G. R. Peterson, and S. M. Meyers, Appl. Phys. Lett. 78, 76 (2001).

${ }^{14}$ S. Zamir, B. Meyler, and J. Salzman, Appl. Phys. Lett. 78, 288 (2001).

${ }^{15}$ P. R. Hageman, S. Haffouz, V. Kirilyuk, A. Grzegorczyk, and P. K. Larsen, Phys. Status Solidi A 188, 523 (2001).

${ }^{16}$ M. H. Kim, Y. Gu Do, H. Choi Kang, D. Y. Noh, and S. J. Park, Appl. Phys. Lett. 79, 2713 (2001).

${ }^{17}$ H. Marchand, J. Appl. Phys. 89, 7846 (2001).

${ }^{18}$ E. Feltin, B. Beaumount, M. Laugt, P. D. Mierry, P. Vennegues, H. Lahhreche, M. Leroux, and P. Gibart, Appl. Phys. Lett. 79, 3230 (2001).

${ }^{19}$ J. Bläsing, A. Reiher, A. Dadger, A. Diez, and A. Krost, Appl. Phys. Lett. 81, 2722 (2002).

${ }^{20}$ J. D. Brown, R. Borges, E. Piner, A. Vescan, S. Singhal, and R. Therrien, Solid-State Electron. 46, 1535 (2002).

${ }^{21}$ S. H. Jang and C. R. Lee, J. Cryst. Growth 253, 64 (2003).

${ }^{22}$ A. Dadgar, M. Poschenrieder, J. Bläsing, O. Contreras, F. Bertram, T. Riemann, A. Reiher, M. Kunze, I. Daumiller, A. Krtschil, A. Diez, A. Kaluza, A. Modlich, M. Kamp, J. Christen, F. A. Ponce, E. Kohn, and A. Krost, J. Cryst. Growth 248, 556 (2003).

${ }^{23}$ S. Raghvan and J. M. Redwing, J. Appl. Phys. 98, 023514 (2005).

${ }^{24}$ C. Mo, W. Fang, Y. Pu, H. Liu, and F. Jiang, J. Cryst. Growth 258, 312 (2005).

${ }^{25}$ M. Dudley, Mater. Res. Soc. Symp. Proc. 892, 0892-FF26-01 (2005).

${ }^{26}$ K. B. Nam, J. Li, M. L. Nakarmi, J. Y. Lin, and H. X. Jiang, Appl. Phys. Lett. 82, 1694 (2003).

${ }^{27}$ J. Li, K. B. Nam, M. L. Nakarmi, J. Li, J. Y. Lin, H. X. Jiang, P. Carrier, and S. H. Wei, Appl. Phys. Lett. 83, 5163 (2003).

${ }^{28}$ S. Arulkumaran, M. Sakai, T. Egawa, H. Ishikawa, T. Jimbo, T. Shibata, K. Asai, S. Sumiya, Y. Kuraoka, M. Tanaka, and O. Oda, Appl. Phys. Lett. 81, 1131 (2002).

${ }^{29}$ B. Zhang, T. Egawa, H. Ishikawa, Y. Liu, and T. Jimbo, J. Appl. Phys. 95, 3170 (2004).

${ }^{30}$ K. H. Kim, Z. Y. Fan, M. Khizar, M. L. Nakarmi, J. Y. Lin, and H. X. Jiang, Appl. Phys. Lett. 85, 4777 (2004). 